\title{
Challenges facing islet transplantation for the treatment of type 1 diabetes mellitus
}

\author{
Kristina I. Rother and David M. Harlan
}

Islet and Autoimmunity Branch, National Institutes of Diabetes and Digestive and Kidney Diseases, NIH, Bethesda, Maryland, USA.

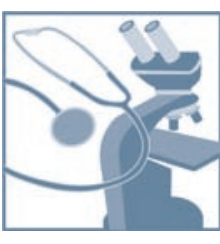

Islet transplantation represents a most impressive recent advance in the search for a type 1 diabetes mellitus cure. While several hundred patients have achieved at least temporary insulin independence after receiving the islet "mini-organs" (containing insulin-producing $\beta$ cells), very few patients remain insulin independent beyond 4 years after transplantation. In this review, we describe historic as well as technical details about the procedure and provide insight into clinical and basic research efforts to overcome existing hurdles for this promising therapy.

Worldwide, more than 750 individuals with type 1 diabetes mellitus (T1DM) have received allogeneic islet transplants since 1974, in an effort to cure their chronic condition. Though this is still a small number (especially when compared with the estimated 1 million afflicted with T1DM and an additional 17 million with type 2 diabetes in the US, not to mention the estimated 140 million with diabetes worldwide), much has been learned, especially since the promising results of the Edmonton group were published in $2000(1,2)$. This report described 7 consecutive patients with T1DM who became insulin independent after receiving islet allografts, which reflects a success rate never previously achieved. The initial enthusiasm over the observation that islet transplantation can restore insulin-independent euglycemia to patients with long-standing T1DM has been dampened by complications associated with the procedure itself and the immunosuppression necessary to prevent rejection of the transplanted islets, as well as by the gradual loss of islet function and other problems arising from the placement of allogeneic islets in the liver $(3,4)$.

At the same time, our understanding about the natural history of T1DM has changed. Epidemiological data indicate that the prognosis for survival among patients with T1DM is good and improving (5-7). Additional evidence strongly suggests that a significant minority with even long-standing T1DM continue to display islet function (8). For instance, we found that at least $40 \%$ of individuals with chronic T1DM (mean duration of 23 years) screened for our islet transplantation protocol had measurable circulating C-peptide levels, an equimolar by-product of endogenous insulin production (B.J. Digon et al., unpublished results). Similar percentages of patients with persistent $\mathrm{C}$-peptide secretion have been documented in the literature (9-11), contrasted by a smaller number of individuals (11\%) reported in the Diabetes Control and Complications Trial (12). Further, old (13) as well as more recent sporadic case reports (14) and other small studies (15) have suggested that diabetes can sometimes resolve and/or that pancreatic insulin production can at least be promoted in patients with long-

Nonstandard abbreviations used: ES cell, embryonic stem cell; IBMIR, instant blood-mediated inflammatory reaction; T1DM, type 1 diabetes mellitus.

Conflict of interest: The authors have declared that no conflict of interest exists. Citation for this article: J. Clin. Invest. 114:877-883 (2004). doi:10.1172/JCI200423235.

The Science in medicine series is supported in part by a generous grant from the Doris Duke Charitable Foundation. standing T1DM. These studies have raised heretofore underexplored avenues for clinical investigation, which we will return to.

\section{Brief history}

In 1924, after approximately 40 years of unsuccessful attempts by various investigators to control diabetes using partial pancreas transplantation, the English surgeon Charles Pybus (1882-1975) made a statement that resonates even today: "Not much can be said about the principles of grafting, but it seems that until we are able to understand them (and I feel we do not understand them at present, especially the chemical factors), then we must continue to fail in such operations, although they may appear the most rational treatment for the diseases for which they are attempted" $(16,17)$. Almost 50 years later, Ballinger and Lacy reported their results isolating and transplanting islets into rats (18). In the late 1970s, various groups including Najarian, Sutherland, et al. (19), and Largiader et al. (20) described their experience with intraportal and intrasplenic human islet allotransplants in patients with non-autoimmune diabetes, one of which was successful for at least a 10 -month follow-up period. In 1990 Scharp et al. reported similar success in a patient with T1DM (21); their results were made possible in part by improved islet isolation techniques developed by Ricordi and colleagues (22). It was, however, only after the Edmonton group's report (2) that islet transplantation appeared as a true alternative to conservative medical management or whole-organ pancreas transplantation.

The Edmonton report stimulated several medical centers worldwide to rejuvenate or establish islet transplant facilities. However, islet transplantation efforts have confronted logistical limitations including the costs associated with cadaveric pancreas procurement, the supplies and equipment required to perform islet isolation, and an experienced team with the relevant expertise in cell processing and invasive radiology, as well as general medicine and nursing. However real these mundane fiscal constraints may be, several other and rather significant biological hurdles currently being addressed on several fronts also limit the field. The remainder of this review will focus upon those hurdles and ongoing research efforts to overcome them.

\section{Supply of cells capable of physiologically regulated insulin secretion}

As of today, the only cells known to be capable of sensing a human being's ambient blood glucose and converting that information into appropriately regulated insulin secretion are the $\beta$ cells found 
in intact islets of Langerhans. As a source of physiologically regulated insulin, $\beta$ cells can be transplanted as the small minority of cells present within a whole pancreas (as is currently performed by wholeorgan pancreas transplantation) or as the enriched fraction of cell clusters we call "isolated islets." Two points are worth emphasizing with regard to isolated islets. First, all such preparations contain significant if variable amounts of contaminating vascular, ductal, and/or acinar tissue despite the purification procedure. The effect of such impurities on patient outcome is not known; however, no adverse reactions to non-islet transplanted tissue have been described to date. Second, isolated islets are best described as mini-organs in that they are highly organized cell clusters with intricate paracrine crosstalk among insulin-producing $\beta$ cells, glucagon-producing $\alpha$ cells, and other hormone-producing cells (23). Islets also have a rich capillary network (24) and contain resident cells with presumed immunological function. Regardless how islets are "packaged" for transplant, i.e., within a pancreas for a vascularized whole-organ graft or as an isolated islet preparation, for all practical purposes, the source must be a brain-dead donor, and the number of such potential donors is quite limited. After unsuitable organ donors are eliminated (e.g., those suffering from malignant or infectious diseases or others with sudden cardiovascular collapse and unacceptable warm ischemia time, which is associated with a rapid decline of organ function), only about 12,000 individuals in the United States remain as potential organ donors each year. However, despite considerable time, effort, and money directed at improving the donation rate, family consent for donation is given only about half the time. Thus, only approximately 6,000 human pancreata are available in the United States each year for transplantation. Further exacerbating the problem, human islet isolation efforts successfully yield preparations with sufficient islet numbers of suitable quality only about half the time; thus current practice could yield approximately 3,000 transplantation-quality preparations per year. Last, nearly all published islet transplantation experience suggests that for most recipients, islets from 2-4 donors are required in order to promote the engraftment of sufficient insulin-producing cells to achieve insulin independence. Most islet recipients have been selected in part due to their small physical size and normal insulin sensitivity: they require fewer donor islets, which increases the likelihood that the limited islet mass transplanted will confer insulin independence $(25,26)$. If patients with greater body mass indices and/or with insulin resistance were also considered for an islet transplant, the 3,000 transplantable islet preparations presently achievable would likely be sufficient to restore euglycemia to fewer than 1,000 patients per year, or less than $0.1 \%$ of patients with T1DM, or approximately $0.005 \%$ of those with either form of diabetes.

\section{Alternative sources of cells with physiologically regulated insulin secretion}

Other than cadaveric pancreata, many potential cellular sources for physiologically regulated insulin secretion have been widely discussed in the scientific literature, but the chasm between the promise each holds and practical reality remains quite broad. Three potential sources in particular have been widely discussed: "growing" islets in vitro; using islets from species other than humans; and promoting $\beta$ cell differentiation from precursor stem cells.

Expanding islet cellular mass in vitro. While a variety of culture conditions have been published for propagating islet cells from adult donors, those techniques invariably result in an inexorable decline in insulin production by the cultured cells (see refs. 27-29). Stated another way, while many groups have reported a favorable stoichiometry with regard to islet cell numbers, no group has developed a strategy for reliably expanding islet populations with dependable and robust insulin-producing capacity. Further, as islets are essentially mini-organs, in order for propagation to occur in vitro, an islet (or the appropriate precursor cells within the islet) must presumably - in no particular order - divide, leave the original islet, then reaggregate with other appropriate cells to create a new islet, while leaving the original mini-organ structure sufficiently intact to remain functional. Last, it is not yet possible to identify the cell(s) within the islet preparation that are responsible for new islet growth. Several reports have suggested that islet progenitor cells exist within the pancreatic ducts (30-32), and yet a recent publication cogently argues that the only cell capable of differentiating into a $\beta$ cell in the adult mouse is an existing $\beta$ cell (33).

Islets from species other than bumans. Others have studied a xenogeneic source for the desired physiologically regulated insulin-secreting cell. Pig islets, in particular, have been widely studied for a variety of reasons, including: (a) the fact that humans had been treated with pig insulin for more than 60 years; (b) favorable husbandry - in that the species has large litters with offspring that attain adult size rapidly and with relatively robust islet numbers; (c) the fact that pig islets respond to glucose in the same physiological glucose range as human islets; and (d) the existence of a suitable societal-cultural relationship between the species. That is, since pigs are currently widely bred, then slaughtered for our food supply, the use of their islets to restore health may be an option that satisfies ethical concerns in a more widely accepted way than would, for example, islets isolated from dogs. Two main concerns continue to limit the use of pig tissues as the xenogeneic source for transplant, however. First, humans express high titers of antibodies against a galactose $\alpha(1$, 3 ) galactose residue that is present on most pig cells, resulting in a hyper-acute rejection response whenever pig tissues are transplanted into humans. Second, pig cells, like all mammalian cells, contain endogenous retrovirus. There is also the possibility that the pig endogenous retrovirus might infect the human host, especially under the circumstance of administration of a large inoculum (as might occur when transplanting tissue) into a host with a weakened immune system (as would be expected when administering immunosuppressive drugs to prevent graft rejection). In order to address these concerns, some investigators have created genetically modified pigs that do not express the galactose $\alpha(1,3)$ galactose residue (34) and plan to breed these animals in special pathogen-free conditions in an effort to minimize the risk for zoonotic disease (35-39).

Promotion of $\beta$ cell differentiation from stem cells. A third, widely studied potential source for insulin-producing cells is a strategy aimed at promoting the differentiation of a precursor cell into a " $\beta$-like cell," with the embryonic stem cell (ES cell) being perhaps the most promising precursor candidate. Clearly, as all mammals generate all cell types from the originally fertilized egg, ES cells are known to have pluripotent capacity. Further, groups have published culture techniques promoting differentiation of ES cells into what appear to be insulin-producing cell clusters $(40,41)$. More recent studies have suggested that at least some of these anti-insulin antibodystaining cell clusters may be artifactual (a result of insulin uptake from the growth media) and that in any case such cultured cell clusters fall short of $\beta$ cells; e.g., they lack insulin granules and the appropriate capacity for regulated insulin secretion (42). Still other research has suggested that additional adult progenitor cell populations can, under some circumstances, differentiate into functional $\beta$ cells in rodents $(43,44)$. Thus, studies by internationally recog- 
Table 1

Systemic side effects commonly associated with the immunosuppressive agents typically administered following islet transplant

Immunosuppressant

(brand name)

Rapamycin, also known as Sirolimus (Rapamune)

FK506, also known as Tacrolimus (Prograf)

Daclizumab

(Zenapax)
Drug classification

Macrocyclic lactone

Calcineurin inhibitor

mAb-binding IL-2 receptor $\alpha$ subunit
Common and important side effects

(Phase of drug administration)

Hyperlipidemia, antiproliferation (e.g., anemia, diarrhea)

(Maintenance)

Hypertension, nephrotoxicity, CNS effects (e.g., tremor), diabetogenicity

(Maintenance)

May increase risk of infections; hypersensitivity

(Induction) nized investigators have yielded data that is difficult to synthesize; i.e., some data suggest that $\beta$ cells in mice can arise only from existing $\beta$ cells, while others suggest a capacity for stem cell-to- $\beta$ cell transdifferentiation (see refs. 33, 43, 44). The outcome of this scientific debate will become evident through additional study.

Several additional points are relevant to the islet supply question; for instance:

(a) If stem cells capable of differentiating into functional $\beta$ cells exist in vivo (in the pancreas, the bone marrow, the spleen, or elsewhere), then what limits the natural occurrence of that process? Why, for instance, do patients with T1DM so rarely, if ever, recover from the loss of $\beta$ cell function that caused their disease?

(b) Why do functional islets form only in islets of Langerhans and the latter only in the pancreas? If our goal is to recapitulate in vitro the process that promoted islet development in vivo, then what hormonal, cell-, and/or matrix-mediated structural signals are generated within the pancreas that promote islet development?

(c) As in all situations where demand outstrips supply, one way to more efficiently utilize the precious resource would be to limit islet loss during isolation or following transplantation. Nilsson, Korsgren, Bennet, et al., for instance, have reported several studies indicating that purified islets, when mixed with whole blood, result in activation of platelets and of both the coagulation and complement cascades in a process they named the instant blood-mediated inflammatory reaction (IBMIR) (45-48). Their studies suggest that IBMIR leads not only to islet destruction (albeit incomplete), but also to activation of an anti-islet adaptive immune response by promoting an innate immune response. Further, IBMIR may contribute to the bleeding and/or thrombotic complications associated with the islet transplant procedure (see below); and

(d) Any insulin-producing cell generated from progenitors in vitro will have to overcome rather stringent regulatory hurdles with regard to human safety. That is, regulatory agencies exist to help navigate the difficult waters that lie between a potential new therapy's initial early successes and the dissemination of that therapy for more widespread application. For instance, when short stature was treated with growth hormone isolated from human pituitary glands, the therapy was shown to be effective but also later to carry with it some risk for Creutzfeldt-Jakob disease. Similarly, we must recognize that cells engineered for physiologically regulated insulin secretion might yield not only the desired consequence once transplanted (insulin independence), but also undesired consequences. For example, rodent studies have suggested that islets transplanted into the liver display an increased propensity to develop into insulinproducing tumors (48), and serious concerns about the role of stem cells in malignancy have been raised (49).

\section{Immunosuppression}

The publication of the Edmonton islet transplantation study (2) addressed an important scientific question: is there a reasonable likelihood that, through transplantation of a sufficient number of islets (see above) and with an immunosuppressive regimen that avoids the use of diabetogenic glucocorticoids, patients will be restored to insulin-independent euglycemia, at least temporarily? For a therapy as expensive and invasive as islet transplantation to be considered for more widespread clinical application, however, an endpoint more rigorous than insulin independence at 1 year after transplant needs to be met. Most important, what do current studies suggest regarding the impact of islet transplantation on patient survival and quality of life?

As shown in Table 1, several systemic side effects are commonly associated with the typically administered immunosuppressive agents (rapamycin and FK506) $(4,50)$, including pneumonitis (51), suppression of bone marrow function, mouth ulcers, deteriorating renal function, peripheral edema, tremor, hyperlipidemia, hypertension, weight loss, diarrhea, and fatigue. While several of these side effects can be countered medically (e.g., hyperlipidemia, hypertension, or infection), the small but significantly increased malignancy risk and the deteriorating renal function require special comment.

T1DM-associated mortality has been associated with several clinical variables; most notably, the traditional cardiovascular risk factors (smoking, blood pressure, lipid levels), autonomic neuropathy, and the degree of renal dysfunction. For the traditional cardiovascular risk factors, while glycemia control decreases microvascular complication rates, no large prospective study has shown a statistically significant effect on macrovascular complications or survival (52, 53). On the other hand, studies have demonstrated that both blood pressure control $(54,55)$ and lipid control $(56)$ decrease diabetesassociated mortality with both high statistical and clinical relevance. However, while autonomic dysfunction has been widely discussed as predictive for diabetes prognosis, more recent and prospective studies have concluded that the apparent association is explained by other factors $(57,58)$. Last, and most important, kidney dysfunction has consistently been found to independently predict mortality among patients with diabetes (59-61). Thus, the increasingly appreciated risk of chronic renal failure after an other-than-kidney transplant (estimated incidence as high as 21\%) (62-64), presumably accounted for at least in part by the calcineurin phosphatase inhibitors - currently the mainstay of most immunosuppressive regimens - has raised concerns regarding the overall impact on survival of a transplant-based approach to the treatment of a diabetic patient. That is, the net effect of improved glycemia control produced by the transplant, when balanced against the immunosuppressive 
agent-associated hypertension, hyperlipidemia, and decreased renal function, may actually increase mortality. Indeed, our recent evaluation of the US pancreas transplant population has suggested statistically greater mortality for at least the first 4 years after transplant for those individuals with preserved kidney function compared with patients with chronic diabetes that is similarly difficult to control but who have not received a transplant (65). Clearly, a critical need exists for strategies to prevent the rejection of cell-based insulin delivery vehicles (isolated islets, whole pancreas, or other strategies) such that kidney function is not impaired. Perhaps antibody-based approaches that are designed to promote anti-graft immune tolerance and therefore minimize the need for chronic administration of immunosuppressive agents (e.g., as in recent studies reported by Herold et al.; refs. 66, 67) will fill this void.

Immunosuppressed organ transplant recipients are also known to be at greater risk for both cancer (relative risk: 3-4) and infection (for instance, see ref. 68) as representative of many studies indicating that well over half of immunosuppressed kidney allograft recipients suffer infectious complications - fortunately almost always easily treated. Among cancers, some relatively rare types predominate in organ transplant recipients as compared with the general population, including lymphomas and lymphoproliferative disorders, Kaposi sarcoma, renal carcinomas, cervical carcinomas in situ, hepatobiliary carcinomas, anogenital carcinomas, and various other sarcomas. In contrast, most of the common malignancies, except skin and lip cancers (69), occur with only marginally increased incidence in immunosuppressed patients compared with nonimmunosuppressed individuals. The risk of developing cancer or infection as a result of the newer antibody-based regimens remains unknown (70).

It is somewhat ironic that current immunosuppressive agents are themselves associated with insulin resistance and/or decreased $\beta$ cell function and as such promote diabetes onset. The incidence of newonset diabetes in organ transplant recipients ranges between $2 \%-53 \%$ (71). The particular immunosuppressive agent employed appears to influence rates of islet engraftment and survival and/or insulin production and action. The diabetogenic effects of glucocorticoids have been the most widely appreciated, in fact since the early descriptions of Cushing syndrome $(72,73)$. Calcineurin phosphatase inhibitors also clearly interfere with normal $\beta$ cell function (74-76). While FK506 appears to confer greater diabetogenic risk than cyclosporin, that observation must be weighed against its preferred use by many transplant teams to prevent allograft loss. Last, studies in SpragueDawley rats have demonstrated that rapamycin, with its known antiproliferative properties, is also associated with insulin resistance (77) and when combined with FK506 (which serves to decrease $\beta$ cell function) induces diabetes. In our experience and according to similar data reported from the Edmonton group (50), patients achieving insulin independence following an islet transplant nevertheless displayed deficient first-phase insulin secretory responses (10\%-30\% of normal) in intravenous glucose tolerance tests. Insulin secretion in such patients is also delayed and deficient after an oral glucose load - reminiscent of responses seen in type 2 diabetes. Clearly, one cannot easily dissect the contribution of each possibly responsible factor such as immunosuppression, abnormal islet innervation and vascularization (24), hepatic localization (78-80), engraftment of a subnormal islet number, and others. We have, however, observed that islet recipients in whom islet function was measurable (from circulating C-peptide) yet insufficient to render them insulin independent had declining glucose control with elevated immunosuppressive drug levels (Rother et al., unpublished results). These data suggest that higher concentrations of immunosuppressive agents can further impair islet function when that function is already challenged. However, with the introduction of novel immunosuppressive regimens, we may be able to avoid such diabetogenic effects.

\section{Safety concerns with the islet transplant procedure}

While almost certainly safer than whole-organ pancreas transplantation, the islet transplant procedure as it currently stands is not risk free. We must remember that while the procedure is not yet deemed a suitable replacement for the current standard treatment for the majority of patients with T1DM, there exists much hope for and possibility of improved efficacy and safety. Some complications must be addressed further. A recent review from the Edmonton group, for instance, reported on complications associated with 68 consecutive islet infusion procedures (81), some of which are potentially serious. These include partial portal vein thrombosis with subsequent anticoagulation (the latter resulting in an expanding hepatic hematoma in 1 patient that required surgery) and/or significant intra-abdominal hemorrhage. Their complication rate can be reported using procedure number as the denominator, in which case the prevalence was $9 \%$ per procedure; or, in recognition of the fact that most patients require more than 1 islet infusion (in the Edmonton report, the mean number of procedures was 2 for the 34 patients reported), the rate of potentially serious complications can be reported as $18 \%$ per patient.

As with any transplantation procedure, although the risk is small, infectious agents can be transmitted via an allograft. An illustration of this point is that despite the extensive screening applied to all blood donors, occasional recipients develop a bloodborne illness from transfusion. Organ and/or tissue donors are extensively screened using known serologic tests, but very recent infections can certainly escape detection.

Long-term safety concerns. Additional safety concerns require consideration, since long-term follow-up of islet recipients remains quite limited. For instance, we and others have observed hepatic structural changes following islet transplantation in both nonhuman primates $(79)$ and in patients $(78,80)$. Both glycogen accumulation and localized steatosis have been demonstrated immunohistochemically and/or by chemical shift MRI. No longterm adverse effects of the transplant procedure on liver function have been reported, but the association between fatty liver disease, obesity, and type 2 diabetes leading to progressive fibrosis and eventual cirrhosis is sobering (82). In fact, it has been speculated that secondary hepatic effects can be used as indirect determinants of islet function, as a recent report described resolution of hepatic imaging abnormalities when the islet allografts failed (78).

Gradually increasing portal venous pressures following islet infusions have also been reported, with each subsequent infusion leading to a greater pressure increase than the last (83). Again, the clinical impact of these pressure increases is unknown.

Finally, while current immunosuppressive approaches are superior to past therapies, both immediate and chronic rejection of transplanted organs and tissues persist as major problems. Since iatrogenic immunosuppression does not completely eliminate the body's immune responses, the remaining mitigated immune activity not only threatens the transplanted organ or tissue but can sensitize the recipient to donor tissues and thus complicate the search for a suitable donor match for any subsequently required transplant. Such sensitization following islet allograft rejection has recently been reported (84). We therefore 


\section{Table 2}

The most important criteria upon which islet transplant recipients have been chosen for existing clinical protocols

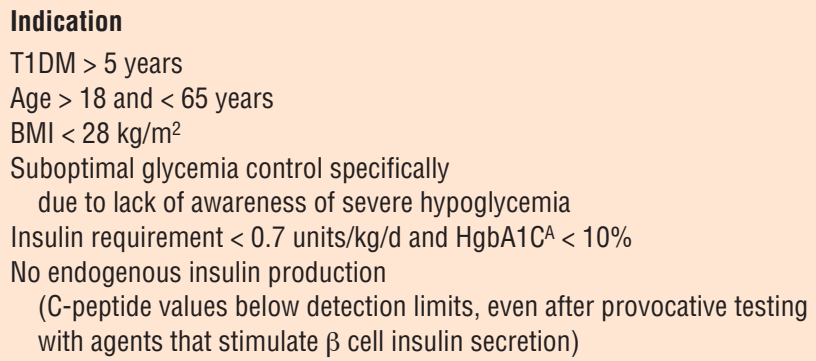

\section{Contraindication}

Cardiac disease

Active infection

Liver abnormalities (e.g., ultrasound evidence of portal hypertension)

Any history of malignancy except squamous or basal skin cancer

Creatinine clearance $<60 \mathrm{ml} / \mathrm{min} / \mathrm{m}^{2}$, or macroalbuminuria

Untreated proliferative retinopathy

Untreated hyperlipidemia

Previous transplant or serologic evidence of

anti-donor tissue specific Ab's

${ }^{A} \mathrm{HgbA1C}$ is the measure of glycosylated hemoglobin that is used to provide an estimate of the average blood glucose control for the previous 3 months.

strongly recommend carefully screening of islet-transplant candidates and exclusion of individuals with early kidney damage from this as-yet-experimental procedure.

\section{Selecting the ideal candidate: victims of our own success}

In a certain sense, improvements in diabetes care have made more difficult the design of clinical trials to overcome the biological hurdles still limiting islet transplantation. The prognosis for patients with even long-standing and brittle T1DM is better than for any other condition treated by transplantation (65), and that prognosis appears to be steadily improving (5-7). Patients with brittle diabetes and end-stage kidney disease may be the best candidates, since the benefits of kidney transplantation in this population have been clearly demonstrated (65); they will thus require immunosuppressive agents to preserve their kidney allograft - adding an islet allograft to the therapeutic mix would seem appropriate. Other patients with brittle diabetes - even those with preserved kidney function - who are plagued by severe hypoglycemia despite optimized care, may also be good candidates (85), but such patients are quite rare, since optimized conservative care frequently eliminates the worst of these symptoms (86). Further, the perception of the term "optimized care" can vary widely, and we urge referral of individuals with diabetes that is difficult to control to specialists well versed in multidisciplinary and the most up-to-date insulin-based regimens before concluding that any patient's diabetes is uncontrollable. We do not support considering individuals with progressive microvascular complications (especially nephropathy) as good candidates due to the known nephrotoxicity of current immunosuppressive regimens. For the same reason, we counsel against islet transplantation in children until more is known about the longterm safety of this approach.

\section{Defining clinical success}

As our intent with this review is to identify those barriers still limiting the more widespread application of islet transplantation for the treatment of diabetes, we wish to emphasize our support for this therapeutic approach. That said, one of the largest hurdles limiting development of this therapy is the difficult interplay of the factors described above. In any experimental technique, one attempts to control all variables except the one under investigation. For the patient given an islet transplant, however, many factors can affect the outcome, with the relative contribution from those disparate variables impossible to tease out. For instance, present technology does not allow investigators to assess with any degree of accuracy the factors that might affect islet function, including: islet quality prior to transplant, the autoimmune response that initially caused the T1DM, the alloimmune response, immunosuppressive effects on islet function, islet dysfunction that might result from metabolic demands placed upon a limited islet mass, etc. Furthermore, when islets are infused into the portal vein, their widespread and diffuse distribution renders impractical any attempt to follow an anti-islet immune response using current biopsy techniques. Thus, if an investigator wishes to test, for example, a novel immunomodulatory approach to prevent islet allograft rejection, and islet function is lost after transplant, it is not yet possible to understand the mechanisms underlying that failure. The many unknowns, considered within the context of the great costs associated with islet transplantation and the relatively excellent prognosis now associated with even long-term diabetes, conspire to create a difficult path for future clinical investigation that falls within well-established medical, ethical, and economical guidelines.

\section{The future}

How and why then do we as a community continue in our pursuit of islet transplantation as a cure for T1DM? Quoting John F. Kennedy's speech given at Rice University in 1962 when he announced the government's plan to land a man on the moon, we choose to pursue islet transplantation research "not because [it is] easy, but because [it is] hard, because that goal will serve to organize and measure the best of our energies and skills, because that challenge is one that we are willing to accept, one we are unwilling to postpone, and one which we intend to win ..." These inspiring words must be considered in the context of what we learn every day from those with T1DM: despite improvements in care, the disease still takes a terrible toll on quality of life.

To be more specific, we suggest the following as potential avenues of research that should be further pursued in the continued development of this most promising therapy. First, we believe an appropriately safe and effective means of preventing immune system-mediated islet destruction after transplant needs to be found. Toward this end, we suggest the following:

(a) While criteria for patient enrollment employed in several protocols evaluating the safety and efficacy of islet transplantation are shown in Table 2, we advocate efforts to define a cohort with highrisk diabetes, as such patients (currently difficult to identify) would 
better fulfill the requirements of appropriate risk/benefit analyses in the evaluation of newer islet transplantation approaches.

(b) Another approach might be to test novel immunomodulatory strategies in, for example, individuals receiving a kidney transplant due to renal failure. Such a population not only has a worse prognosis for survival than do patients with diabetes (and so a greater tolerance for the new immune intervention's potential toxicity), but following the outcome of such patients is much easier and more objective. That is, patients without an autoimmune etiology underlying their renal failure can be selected, a kidney from a single donor is sufficient to treat the patient (whereas islet recipients typically require islets from 2 or more donors), kidney biopsies to follow graft function are now routine, techniques for overcoming an acute kidney rejection episode exist, and donor kidney function can reasonably be assessed. If a novel immune therapy proves sufficiently safe and effective in the kidney transplant recipient population, then such a strategy could be rapidly tested in a selection of islet allograft recipients. As a first step, patients with kidney failure from T1DM and thus requiring a kidney transplant could also receive allogeneic islets.

(c) Still another approach might be to test a novel immunomodulatory regimen in patients with T1DM of some duration yet who still have some measurable level of $\beta$ cell function. As discussed herein, a surprising number of patients with T1DM display some evidence of either an anti- $\beta$ cell immune response (at least antiislet antibody titers) and/or $\beta$ cell function (detectable levels of circulating C-peptide) even years after diagnosis. These observations suggest the as-yet-untested hypothesis that through control of the autoimmune response, and perhaps other factors, some $\beta$ cell functional recovery could ensue (87). An advantage of such an approach is that it would be more scientifically rigorous with less uncontrollable variables (like allogeneic islet quality and alloimmune responses that are difficult to monitor) and also achieves a more supportable risk/benefit balance than current immunosuppression-based protocols that enroll patients with new-onset disease. That is, the testing of an immune intervention of unknown long-term safety in an adult with long-standing T1DM to see whether the $\beta$ cell function might improve seems to fulfill the high standards of clinical investigation in a more balanced fashion than would testing that agent in a patient with new-onset disease (typically a child). We are actively engaged in testing this hypothesis.

(d) We recommend research to develop practical tools for the immunological monitoring of the islet recipient so that both alloand autoimmune responses can be followed.

Second, before we can hope to "cure" patients with diabetes by transplanting cells capable of physiologically regulated insulin secretion, we need a reliable and preferably renewable source of such cells. In general, this means we will need: control of the material and methods required to generate such cells; and reliable assays for determining the cells' quality (viability, insulin producing capacity or "potency," genetic stability, etc.).

At present, allogeneic islets isolated from cadaveric donors fall short of this appropriately high standard, but by clearly recognizing and stating the problems, investigators in the field can focus on overcoming them. The use of islets from cadaveric pancreata will always be hampered by lack of control over the starting material and also remains constrained by their limited supply, the imperfect means of isolating those islets, and the great expense associated with that exercise. The ability to propagate, in vitro or in a surrogate animal species, cells capable of physiologically regulated insulin secretion would be a major step forward.

Last, we advise careful follow-up of those patients already given allogeneic islets by transplant such that many variables are monitored, including the function of those allogeneic islets but also effects the islets may have on liver structure or function, effects of the immunosuppressive agents of diabetes complications, survival, and quality of life. The first annual report of the Collaborative Islet Transplant Registry (see http://spitfire.emmes.com/study/isl/reports/reports.htm) is an important step forward. We also encourage longer-duration studies using large-animal models to address some of these questions.

Address correspondence to: David M. Harlan, Islet and Autoimmunity Branch, National Institutes of Diabetes and Digestive and Kidney Diseases, NIH, Building 10, Room 8N307, Bethesda, Maryland 20892, USA. Phone: (301) 594-3407; Fax: (301) 480-4518; E-mail: davidmh@intra.niddk.nih.gov.
1. Hirshberg, B., Rother, K.I., Digon, B.J., III, Venstrom, J., and Harlan, D.M. 2003. State of the art: islet transplantation for the cure of type 1 diabetes mellitus. Rev. Endocr. Metab. Disord. 4:381-389.

2. Shapiro, A.M., et al. 2000. Islet transplantation in seven patients with type 1 diabetes mellitus using a glucocorticoid-free immunosuppressive regimen. N. Engl. J. Med. 343:230-238.

3. Hirshberg, B., Rother, K.I., and Harlan, D.M. 2003. Islet transplantation: where do we stand now? Diabetes Metab. Res. Rev. 19:175-178.

4. Hirshberg, B., et al. 2003. Solitary islet transplantation for type 1 diabetes mellitus using steroid sparing immunosuppression: the NIH experience. Diabetes Care. 26:3288-3295.

5. Hovind, P., et al. 2003. Decreasing incidence of severe diabetic microangiopathy in type 1 diabetes. Diabetes Care. 26:1258-1264.

6. Nordwall, M., Bojestig, M., Arnqvist, H.J., and Ludvigsson, J. 2004. Declining incidence of severe retinopathy and persisting decrease of nephropathy in an unselected population of Type 1 diabetes - the Linkoping Diabetes Complications Study. Diabetologia. 47:1266-1272.

7. Nishimura, R., et al. 2001. Mortality trends in type 1 diabetes. The Allegheny County (Pennsylvania) Registry 1965-1999. Diabetes Care. 24:823-827.
8. Palmer, J.P., et al. 2004. C-peptide is the appropriate outcome measure for type 1 diabetes clinical trials to preserve beta-cell function: report of an ADA workshop, 21-22 October 2001. Diabetes. 53:250-264.

9. Lohr, M., and Kloppel, G. 1987. Residual insulin positivity and pancreatic atrophy in relation to duration of chronic type 1 (insulin-dependent) diabetes mellitus and microangiopathy. Diabetologia. 30:757-762.

10. Madsbad, S. 1983. Factors of importance for residual beta-cell function in type I diabetes mellitus. A review. Acta Med. Scand. Suppl. 671:61-67.

11. Eff, C., Faber, O., and Deckert, T. 1978. Persistent insulin secretion, assessed by plasma C-peptide estimation in long-term juvenile diabetics with a low insulin requirement. Diabetologia. 15:169-172.

12. Steffes, M.W., Sibley, S., Jackson, M., and Thomas, W. 2003. Beta-cell function and the development of diabetes-related complications in the diabetes control and complications trial. Diabetes Care. 26:832-836.

13. Williams, P.W. 1894. Notes on diabetes treated with extract and by grafts of sheep's pancreas. BMJ. 2:1303-1304.

14. Karges, B., et al. 2004. Complete long-term recovery of beta-cell function in autoimmune type 1 diabetes after insulin treatment. Diabetes Care. 27:1207-1208.

15. Ilic, S., Jovanovic, L., and Wollitzer, A.O. 2000. Is the paradoxical first trimester drop in insulin requirement due to an increase in C-peptide concentration in pregnant Type I diabetic women? Diabetologia. 43:1329-1330.

16. Benedum, J. 1999. The early history of endocrine cell transplantation. J. Mol. Med. 77:30-35.

17. Pybus, F.C. 1924. Notes on suprarenal and pancreatic grafting. Lancet. 550-551.

18. Ballinger, W.F., and Lacy, P.E. 1972. Transplantation of intact pancreatic islets in rats. Surgery. 72:175-186.

19. Najarian, J.S., et al. 1977. Human islet transplantation: a preliminary report. Transplant. Proc. 9:233-236.

20. Largiader, F., Kolb, E., and Binswanger, U. 1980. A long-term functioning human pancreatic islet allotransplant. Transplantation. 29:76-77.

21. Scharp, D.W., et al. 1990. Insulin independence after islet transplantation into type I diabetic patient. Diabetes. 39:515-518.

22. Ricordi, C., Lacy, P.E., Finke, E.H., Olack, B.J., and Scharp, D.W. 1988. Automated method for isolation of human pancreatic islets. Diabetes. 37:413-420.

23. Flattem, N., et al. 2001. Alpha- and beta-cell responses to small changes in plasma glucose in the conscious dog. Diabetes. 50:367-375.

24. Brissova, M., et al. 2004. Intraislet endothelial cells contribute to revascularization of transplanted 
pancreatic islets. Diabetes. 53:1318-1325

25. Hering, B.J., et al. 2004. Transplantation of cultured islets from two-layer preserved pancreases in type 1 diabetes with anti-CD3 antibody. Am. J. Transplant. 4:390-401.

26. Shapiro, A.M., and Ricordi, C. 2004. Unraveling the secrets of single donor success in islet transplantation. Am. J. Transplant. 4:295-298.

27. Beattie, G.M., et al. 2002. A novel approach to increase human islet cell mass while preserving beta-cell function. Diabetes. 51:3435-3439.

28. Beattie, G.M., Hayek, A., and Levine, F. 2000. Growth and genetic modification of human beta-cells and beta-cell precursors. Genet. Eng. 22:99-120.

29. Hayek, A., and Beattie, G.M. 2002. Alternatives to unmodified human islets for transplantation. Curr. Diab. Rep. 2:371-376.

30. Bonner-Weir, S., et al. 2000. In vitro cultivation of human islets from expanded ductal tissue. Proc. Natl. Acad. Sci. U. S. A. 97:7999-8004.

31. Dutta, S., et al. 2001. PDX:PBX complexes are required for normal proliferation of pancreatic cells during development. Proc. Natl. Acad. Sci. U. S. A. 98:1065-1070.

32. Sharma, A., et al. 1999. The homeodomain protein IDX-1 increases after an early burst of proliferation during pancreatic regeneration. Diabetes. 48:507-513.

33. Dor, Y., Brown, J., Martinez, O.I., and Melton, D.A. 2004. Adult pancreatic beta-cells are formed by self-duplication rather than stem-cell differentiation. Nature. 429:41-46.

34. Kolber-Simonds, D., et al. 2004. Production of alpha-1,3-galactosyltransferase null pigs by means of nuclear transfer with fibroblasts bearing loss of heterozygosity mutations. Proc. Natl. Acad. Sci.U.S. A. 101:7335-7340.

35. Blusch, J.H., Patience, C., and Martin, U. 2002. Pig endogenous retroviruses and xenotransplantation. Xenotransplantation. 9:242-251.

36. Magre, S., Takeuchi, Y., and Bartosch, B. 2003. Xenotransplantation and pig endogenous retroviruses. Rev. Med. Virol. 13:311-329.

37. Quinn, G., et al. 2004. Genotyping of porcine endogenous retroviruses from a family of miniature swine. J. Virol. 78:314-319.

38. Martin, U., et al. 2000. Productive infection of primary human endothelial cells by pig endogenous retrovirus (PERV). Xenotransplantation. 7:138-142.

39. Tucker, A., et al. 2002. The production of transgenic pigs for potential use in clinical xenotransplantation: microbiological evaluation. Xenotransplantation. 9:191-202.

40. Hori, Y., et al. 2002. Growth inhibitors promote differentiation of insulin-producing tissue from embryonic stem cells. Proc. Natl. Acad. Sci. U. S. A. 99:16105-16110.

41. Lumelsky, N., et al. 2001. Differentiation of embryonic stem cells to insulin-secreting structures similar to pancreatic islets. Science. 292:1389-1394.

42. Rajagopal, J., Anderson, W.J., Kume, S., Martinez, O.I., and Melton, D.A. 2003. Insulin staining of ES cell progeny from insulin uptake. Science. 299:363.

43. Ianus, A., Holz, G.G., Theise, N.D., and Hussain, M.A. 2003. In vivo derivation of glucose-competent pancreatic endocrine cells from bone marrow without evidence of cell fusion. J. Clin. Invest. 111:843-850. doi:10.1172/JCI200316502.

44. Kodama, S., Kuhtreiber, W., Fujimura, S., Dale, E.A., and Faustman, D.L. 2003. Islet regeneration during the reversal of autoimmune diabetes in NOD mice. Science. 302:1223-1227.

45. Bennet, W., Groth, C.G., Larsson, R., Nilsson, B., and Korsgren, O. 2000. Isolated human islets trigger an instant blood mediated inflammatory reaction: implications for intraportal islet transplantation as a treatment for patients with type 1 diabetes. Ups. J. Med. Sci. 105:125-133.
46. Johansson, U., Olsson, A., Gabrielsson, S., Nilsson, B., and Korsgren, O. 2003. Inflammatory mediators expressed in human islets of Langerhans: implications for islet transplantation. Biochem. Biophys. Res. Commun. 308:474-479.

47. Moberg, L., et al. 2002. Production of tissue factor by pancreatic islet cells as a trigger of detrimental thrombotic reactions in clinical islet transplantation. Lancet. 360:2039-2045.

48. Dombrowski, F., Klingmuller, D., and Pfeifer, U. 1998. Insulinomas derived from hyperplastic intra-hepatic islet transplants. Am. J. Pathol. 152:1025-1038.

49. Marx, J. 2003. Cancer research. Mutant stem cells may seed cancer. Science. 301:1308-1310.

50. Ryan, E.A., et al. 2002. Successful islet transplantation: continued insulin reserve provides long-term glycemic control. Diabetes. 51:2148-2157.

51. Digon, B.J., III, Rother, K.I., Hirshberg, B., and Harlan, D.M. 2003. Sirolimus-induced interstitial pneumonitis in an islet transplant recipient. Diabetes Care. 26:3191.

52. The Diabetes Control and Complications Trial Research Group. 1993. The effect of intensive treatment of diabetes on the development and progression of long-term complications in insulin-dependent diabetes mellitus. N. Engl. J. Med. 329:977-986.

53. UK Prospective Diabetes Study Group. 1998. Intensive blood-glucose control with sulphonylureas or insulin compared with conventional treatment and risk of complications in patients with type 2 diabetes (UKPDS 33). Lancet. 352:837-853.

54. UK Prospective Diabetes Study Group. 1998. Tight blood pressure control and risk of macrovascular and microvascular complications in type 2 diabetes: UKPDS 38. BMJ. 317:703-713.

55. Adler, A.I., et al. 2000. Association of systolic blood pressure with macrovascular and microvascular complications of type 2 diabetes (UKPDS 36): prospective observational study. BMJ. 321:412-419.

56. Collins, R., Armitage, J., Parish, S., Sleigh, P., and Peto, R. 2003. MRC/BHF Heart Protection Study of cholesterol-lowering with simvastatin in 5963 people with diabetes: a randomised placebo-controlled trial. Lancet. 361:2005-2016.

57. Orchard, T.J., Lloyd, C.E., Maser, R.E., and Kuller, L.H. 1996. Why does diabetic autonomic neuropathy predict IDDM mortality? An analysis from the Pittsburgh Epidemiology of Diabetes Complications Study. Diabetes Res. Clin. Pract. 34(Suppl.):S165-S171.

58. Soedamah-Muthu, S.S., et al. 2004. Risk factors for coronary heart disease in type 1 diabetic patients in Europe: the EURODIAB Prospective Complications Study. Diabetes Care. 27:530-537.

59. Allen, K.V., and Walker,J.D. 2003. Microalbuminuria and mortality in long-duration type 1 diabetes. Diabetes Care. 26:2389-2391.

60. Sarnak, M.J., et al. 2003. Kidney disease as a risk factor for development of cardiovascular disease: a statement from the American Heart Association Councils on Kidney in Cardiovascular Disease, High Blood Pressure Research, Clinical Cardiology, and Epidemiology and Prevention. Circulation. 108:2154-2169.

61. Strippoli, G.F., et al. 2003. Clinical and therapeutic aspects of diabetic nephropathy. J. Nephrol. 16:487-499.

62. Mazur, M.J., et al. 2004. Decline in native renal function early after bladder-drained pancreas transplantation alone. Transplantation. 77:844-849.

63. Ojo, A.O., et al. 2003. Chronic renal failure after transplantation of a nonrenal organ. N. Engl. J. Med. 349:931-940

64. Rubel, J.R., Milford, E.L., McKay, D.B., and Jarcho, J.A. 2004. Renal insufficiency and end-stage renal disease in the heart transplant population. J. Heart Lung Transplant. 23:289-300.

65. Venstrom, J.M., et al. 2003. Pancreas transplantation decreases survival for patients with diabetes and pre- served kidney function. JAMA. 290:2817-2823.

66. Herold, K.C., et al. 2002. Anti-CD3 monoclonal antibody in new-onset type 1 diabetes mellitus. N. Engl. J. Med. 346:1692-1698.

67. Steele, C., et al. 2004. Insulin Secretion in Type 1 Diabetes. Diabetes. 53:426-433.

68. Boggi, U., et al. 2004. A benefit-risk assessment of basiliximab in renal transplantation. Drug Saf. 27:91-106.

69. Penn, I. 2000. Post-transplant malignancy: the role of immunosuppression. Drug Saf. 23:101-113.

70. Couzin, J. 2003. Clinical trials. Diabetes' brave new world. Science. 300:1862-1865.

71. Montori, V.M., et al. 2002. Posttransplantation diabetes: a systematic review of the literature. Diabetes Care. 25:583-592.

72. Davani, B., et al. 2004. Aged transgenic mice with increased glucocorticoid sensitivity in pancreatic beta-cells develop diabetes. Diabetes. 53(Suppl. 1):S51-S59.

73. Delaunay, F., et al. 1997. Pancreatic beta cells are important targets for the diabetogenic effects of glucocorticoids. J. Clin. Invest. 100:2094-2098.

74. Bloom, R.D., et al. 2002. Association of hepatitis $C$ with posttransplant diabetes in renal transplant patients on tacrolimus. J. Am. Soc. Nephrol. 13:1374-1380.

75. First, M.R., Gerber, D.A., Hariharan, S., Kaufman, D.B., and Shapiro, R. 2002. Posttransplant diabetes mellitus in kidney allograft recipients: incidence, risk factors, and management. Transplantation. 73:379-386.

76. Weir, M.R., and Fink, J.C. 1999. Risk for posttransplant Diabetes mellitus with current immunosuppressive medications. Am. J. Kidney Dis. 34:1-13.

77. Lopez-Talavera, J.C., et al. 2004. Hepatocyte growth factor gene therapy for pancreatic islets in diabetes: reducing the minimal islet transplant mass required in a glucocorticoid-free rat model of allogeneic portal vein islet transplantation. Endocrinology. 145:467-474.

78. Bhargava, R., et al. 2004. Prevalence of hepatic steatosis after islet transplantation and its relation to graft function. Diabetes. 53:1311-1317.

79. Hirshberg, B., Mog, S., Patterson, N., Leconte, J., and Harlan, D.M. 2002. Histopathological study of intrahepatic islets transplanted in the nonhuman primate model using Edmonton protocol immunosuppression. J. Clin. Endocrinol. Metab. 87:5424-5429.

80. Markmann, J.F., et al. 2003. Magnetic resonancedefined periportal steatosis following intraportal islet transplantation: a functional footprint of islet graft survival? Diabetes. 52:1591-1594.

81. Owen, R.J., et al. 2003. Percutaneous transhepatic pancreatic islet cell transplantation in type 1 diabetes mellitus: radiologic aspects. Radiology. 229:165-170.

82. Clouston, A.D., and Powell, E.E. 2004. Nonalcoholic fatty liver disease: is all the fat bad? Intern. Med. J. 34:187-191.

83. Casey, J.J., et al. 2002. Portal venous pressure changes after sequential clinical islet transplantation. Transplantation. 74:913-915.

84. Han, D., et al. 2004. Assessment of cytotoxic lymphocyte gene expression in the peripheral blood of human islet allograft recipients: elevation precedes clinical evidence of rejection. Diabetes. 53:2281-2290.

85. Ryan, E.A., et al. 2004. Assessment of the severity of hypoglycemia and glycemic lability in type 1 diabetic subjects undergoing islet transplantation. Diabetes. 53:955-962.

86. Cryer, P.E. 2004. Diverse causes of hypoglycemiaassociated autonomic failure in diabetes. N. Engl. J. Med. 350:2272-2279.

87. Ruggles, J.A., Kelemen, D., and Baron, A. 2004. Emerging therapies: controlling glucose homeostasis, immunotherapy, islet transplantation, gene therapy, and islet cell neogenesis and regeneration [review]. Endocrinol. Metab. Clin. North Am. 33:239-252, xii. 